6 pages, 2 figures

\title{
Non-stationary effects in the coupled quantum dots influenced by the electron-phonon interaction
}

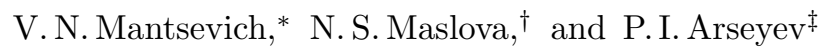 \\ Moscow State University, Department of Physics, \\ 119991 Moscow, Russia \\ P.N. Lebedev Physical institute of RAS, \\ 119991, Moscow, Russia \\ (Dated: August 9, 2021)
}

\begin{abstract}
We analyzed time evolution of the localized charge in the system of two interacting single level quantum dots (QDs) coupled with the continuous spectrum states in the presence of electron-phonon interaction.

We demonstrated that electron-phonon interaction leads to the increasing of localized charge relaxation rate. We also found that several time scales with different relaxation rates appear in the system in the case of non-resonant tunneling between the dots. We revealed the formation of oscillations in the filling numbers time evolution caused by the emission and adsorption processes of phonons.
\end{abstract}

PACS numbers: 73.20.Hb, 73.23.Hk, 73.40.Gk

Keywords: D. Electron-phonon interaction; D. Non-equilibrium non-stationary filling numbers; D. Quantum dots; D. Relaxation

\section{INTRODUCTION}

Recent progress in the engineering and fabrication of well-defined artificial systems - quantum dots (QDs), leads to the possibility of ultra small electronic devices formation with a relatively high control of system parameters (size, shape and energy spectrum) [1]. In addition to the QDs potential industrial applications, these nanoscale objects provide an ideal test ground for the study of basic physics including, many-body interaction effects, electron transport and time-dependent effects. Moreover, QDs integration in a little quantum circuits deals with careful analysis of non-equilibrium charge distribution, relaxation processes and non-stationary effects. These processes influence strongly on the electron transport through the system of QDs [2], [3], [4], [5], [6], [7]. Electron transport in such systems is governed not only by the Coulomb interaction between localized electrons [5], 6], 7] but also by the electron-phonon interaction [8], [9], 10].

Most of the theoretical works devoted to the problem of electron transport through the coupled QDs in the presence of electron-phonon interaction deal with the tunneling current and current-current correlations (shot noise) investigations [10], 11]. Only a few attempts have been made to analyze phonon assisted localized charge relaxation [13], 14], 15]. It was found theoretically that lateral confinement influence on the single-electron relaxation

\footnotetext{
*vmantsev@spmlab.phys.msu.ru

${ }^{\dagger}$ Electronic address: spm@spmlab.phys.msu.ru

$\ddagger_{\text {ars@lpi.ru }}$
}

rates in parabolic QDs [12]. Author considered the deformation potential coupling between electrons and longitudinal acoustic phonons, while neglecting piezoelectric coupling on the grounds of it's weaker contribution in two dimensional structures. On the other hand further theoretical and experimental works suggested that electronacoustic phonon scattering due to the piezoelectric field interaction is relevant for momentum and spin relaxation processes [16], [17] and may even provide charge decoherence in laterally coupled QDs [13], [14]. In [15] authors analyzed phonon induced single electron relaxation rates in the models of weakly confined single and vertically coupled QDs taking into account both mentioned above mechanisms. The regimes where each coupling mechanism prevails were found.

In this paper we use the Keldysh diagram technique [18] to analyze charge relaxation in a double QDs due to the coupling with the continuous spectrum states in the presence of electron-phonon interaction. Tunneling to the reservoir is possible only from one of the dots. We have found that electron-phonon interaction results in the increasing of localized charge relaxation rate and also leads to the formation of well resolved oscillations.

\section{THE SUGGESTED MODEL}

In the present paper we consider a system of coupled QDs with the single particle levels $\varepsilon_{1}$ and $\varepsilon_{2}$ connected with the continuous spectrum states. At the initial time two electrons with opposite spins are localized in the first $\mathrm{QD}$ on the energy level $\varepsilon_{1}\left(n_{1 \sigma}(0)=n_{0}=1\right)$. The second QD with the energy level $\varepsilon_{2}$ is connected with the reservoir $\left(\varepsilon_{p}\right)$. Relaxation of the localized charge is governed 
by the Hamiltonian:

$$
\hat{H}=\hat{H}_{D}+\hat{H}_{t u n}+\hat{H}_{\text {res }}
$$

The Hamiltonian $\hat{H}_{D}$ of interacting QDs

$$
\begin{aligned}
\hat{H}_{D} & =\sum_{i=1,2 \sigma} \varepsilon_{i} c_{i \sigma}^{+} c_{i \sigma}+\sum_{\sigma} T\left(c_{1 \sigma}^{+} c_{2 \sigma}+c_{1 \sigma} c_{2 \sigma}^{+}\right)+ \\
& +\omega_{0} b^{+} b+g\left(c_{1 \sigma}^{+} c_{2 \sigma}+c_{1 \sigma} c_{2 \sigma}^{+}\right)\left(b^{+}+b\right)
\end{aligned}
$$

contains the spin-degenerate levels $\varepsilon_{i}$ (indexes $i=1$ and $i=2$ correspond to the first and to the second QD) and electron-phonon interaction. The creation/annihilation of an electron with spin $\sigma= \pm 1$ within the dot is denoted by $c_{i \sigma}^{+} / c_{i \sigma}$ and $n_{\sigma}$ is the corresponding filling number operator. Operators $b^{+} / b$ describe the creation/annihilation of the phonons. $\omega_{0}$ - is the optical phonon frequency and $g$ - is the electron-phonon coupling constant. The interaction between the dots is described by the tunneling transfer amplitude $T$ which is considered to be independent of momentum and spin.

The coupling between the second dot and the continuous spectrum states is described by the Hamiltonian:

$$
\hat{H}_{t u n}=\sum_{p \sigma} t\left(c_{p \sigma}^{+} c_{2 \sigma}+c_{p \sigma} c_{2 \sigma}^{+}\right),
$$

where $t$ is the tunneling amplitude, which we assume to be independent on momentum and spin. By considering a constant density of states in the reservoir $\nu_{0}$, the tunnel rate $\gamma$ is defined as $\gamma=\pi \nu_{0} t^{2}$.

The continuous spectrum states are modeled by the Hamiltonian:

$$
\hat{H}_{r e s}=\sum_{p \sigma} \varepsilon_{p} c_{p \sigma}^{+} c_{p \sigma}
$$

where $c_{p \sigma}^{+} / c_{p \sigma}$ creates/annihilates an electron with spin $\sigma$ and momentum $p$ in the lead. We shall use Keldysh diagram technique to describe charge density relaxation processes in the considered system. Time evolution of the electron density in the QD is determined by the Keldysh Green function $G_{11}^{<}$which is connected with the localized state filling numbers in the following way:

$$
G_{11}^{<}(t, t)=i n_{1}(t)
$$

Integro-differential equations for Green function $G_{11}^{<T}\left(t, t^{\prime}\right)$ without electron-phonon interaction has the form:

$$
\begin{aligned}
G_{11}^{<T}\left(t, t^{\prime}\right) & =G_{11}^{0<}+G_{11}^{0 R} T^{2} G_{22}^{0 R} G_{11}^{<}+ \\
& +G_{11}^{0 R} T^{2} G_{22}^{0<} G_{11}^{A T}+G_{11}^{0<} T^{2} G_{22}^{0 A} G_{11}^{A T}
\end{aligned}
$$

In the case when initial charge is localized in the first QD and the second dot is empty, the third term in the eq. (6) can be neglected. Retarded Green's function $G_{11}^{A T}\left(t^{\prime}, t\right)=\left[G_{11}^{R T}\left(t, t^{\prime}\right)\right]^{*}$ yields density of states in the first QD and can be found exactly from the integral equation:

$$
G_{11}^{R T}=G_{11}^{0 R}+G_{11}^{0 R} T^{2} G_{22}^{0 R} G_{11}^{R}
$$

where Green's functions $G_{11}^{0 R}\left(t-t^{\prime}\right)$ and $G_{22}^{0 R}\left(t-t^{\prime}\right)$ in the absence of coupling between the dots are determined by the expressions:

$$
\begin{aligned}
& G_{11}^{0 R}\left(t-t^{\prime}\right)=-i \Theta\left(t-t^{\prime}\right) e^{-i \varepsilon_{1}\left(t-t^{\prime}\right)} \\
& G_{22}^{0 R}\left(t-t^{\prime}\right)=-i \Theta\left(t-t^{\prime}\right) e^{-i \varepsilon_{2}\left(t-t^{\prime}\right)-\gamma\left(t-t^{\prime}\right)}
\end{aligned}
$$

The eigenfrequencies $E_{1,2}$ of equation (7) are determined in the following way

$$
\begin{array}{r}
\left(E-\varepsilon_{1}\right)\left(E-\varepsilon_{2}+i \gamma\right)-T^{2}=0 \\
E_{1,2}=\frac{1}{2}\left(\varepsilon_{1}+\varepsilon_{2}-i \gamma\right) \pm \frac{1}{2} \sqrt{\left(\varepsilon_{1}-\varepsilon_{2}+i \gamma\right)^{2}+4 T^{2}}
\end{array}
$$

Finally retarded Green's function can be written as:

$$
\begin{array}{r}
G_{11}^{R T}\left(t, t^{\prime}\right)=-i \Theta\left(t-t^{\prime}\right)\left(\frac{E_{1}-\varepsilon_{2}+i \gamma}{E_{1}-E_{2}} e^{-E_{1}\left(t-t^{\prime}\right)}-\right. \\
\left.-\frac{E_{2}-\varepsilon_{2}+i \gamma}{E_{1}-E_{2}} e^{-E_{2}\left(t-t^{\prime}\right)}\right)
\end{array}
$$

and interaction with the continuous spectrum states is included in the Green's function $G_{22}^{0 R}\left(t-t^{\prime}\right)$. Electronphonon interaction results in the appearance of corrections to the Green's function $G_{11}^{R T}$ in the equations (6) and (7). Consequently the equation for Green function has the following form:

$$
\begin{aligned}
G_{11}^{R}\left(t, t^{\prime}\right) & =G_{11}^{0 R}+G_{11}^{0 R} T^{2} G_{22}^{0 R} G_{11}^{R}+G_{11}^{0 R} \Sigma_{11}^{R} G_{11}^{R}+ \\
& +G_{11}^{0 R} \Sigma_{12}^{R} G_{21}^{R}+G_{12}^{0 R} \Sigma_{21}^{R} G_{11}^{R}+G_{12}^{0 R} \Sigma_{22}^{R} G_{21}^{R}
\end{aligned}
$$

where self-energies $\Sigma_{11}^{R}\left(t, t^{\prime}\right), \Sigma_{12}^{R}\left(t, t^{\prime}\right), \Sigma_{21}^{R}\left(t, t^{\prime}\right)$ and $\Sigma_{22}^{R}\left(t, t^{\prime}\right)$ can be written as:

$$
\begin{aligned}
& \Sigma_{11}^{R}\left(t, t^{\prime}\right)=i g^{2}\left[D^{>} G_{22}^{A T}+D^{R} G_{22}^{<T}\right] \\
& \left.\Sigma_{12}^{R}\left(t, t^{\prime}\right)=i g^{2}\left[D^{>} G_{21}^{A T}+D^{R}\right) G_{21}^{<T}\right] \\
& \Sigma_{21}^{R}\left(t, t^{\prime}\right)=i g^{2}\left[D^{>} G_{12}^{A T}+D^{R} G_{12}^{<T}\right] \\
& \Sigma_{22}^{R}\left(t, t^{\prime}\right)=i g^{2}\left[D^{>} G_{11}^{A T}+D^{R} G_{11}^{<T}\right]
\end{aligned}
$$

In the eq. (12) the following ratio is considered $G_{22}^{A T}\left(t^{\prime}, t\right)=\left[G_{22}^{R T}\left(t, t^{\prime}\right)\right]^{*}$ and expression for Green's 
function $G_{22}^{R T}\left(t, t^{\prime}\right)$ analogous to the equation (10) has the following form:

$$
\begin{array}{r}
G_{22}^{R T}\left(t, t^{\prime}\right)=-i \Theta\left(t-t^{\prime}\right)\left(\frac{E_{2}-\varepsilon_{1}}{E_{1}-E_{2}} e^{-E_{1}\left(t-t^{\prime}\right)}-\right. \\
\left.-\frac{E_{1}-\varepsilon_{1}}{E_{1}-E_{2}} e^{-E_{2}\left(t-t^{\prime}\right)}\right)
\end{array}
$$

The last three terms in eq.(11) correspond to the next order perturbation theory in the parameter $\frac{T^{2}}{\gamma^{2}}$. Consequently, localized charge relaxation in the presence of electron-phonon interaction is mostly governed by the term $G_{11}^{0 R} \Sigma_{11}^{R} G_{11}^{R}$. Acting with operator $G_{11}^{0 R-1}$ and considering the terms with the accuracy $\frac{T^{2}}{\gamma^{2}}$ one can re-write the equation (11) in the following way:

$$
\left(G_{11}^{0 R-1}-T^{2} G_{22}^{0 R}-\Sigma_{11}^{R}\right) G_{11}^{R}\left(t, t^{\prime}\right)=\delta\left(t-t^{\prime}\right)
$$

The eigenvalues of equation (14) can be found from characteristic equation written in the following form:

$$
\begin{array}{r}
{\left[G_{11}^{0 R-1}(\omega) G_{22}^{0 R-1}(\omega)-T^{2}\right]} \\
\cdot\left[G_{11}^{0 R-1}\left(\omega-\omega_{0}\right) G_{22}^{0 R-1}\left(\omega-\omega_{0}\right)-T^{2}\right]- \\
-g^{2} \cdot\left(2 N_{0 \omega}+1\right) \cdot G_{22}^{0 R-1}(\omega) G_{11}^{0 R-1}\left(\omega-\omega_{0}\right)=0
\end{array}
$$

where functions $G_{i i}^{0 R-1}$ can be determined as

$$
G_{i i}^{0 R-1}=i \frac{\partial}{\partial t}-\varepsilon_{i}
$$

Consequently retarded Green's function $G_{11}^{R}$ can be written in the following form:

$$
G_{11}^{R}\left(t, t^{\prime}\right)=\sum_{i}-i \Theta\left(t-t^{\prime}\right) A_{i} e^{-i E_{i}\left(t-t^{\prime}\right)}
$$

where $E_{i}$ - are eigenvalues of equation (15). Coefficients $A_{i}$ can be found from the system of linear equations obtained in the first order perturbation theory in the parameter $g^{2}$ :

$$
\begin{aligned}
\sum_{i=1}^{4} A_{i} & =1 \\
-\sum_{i=1}^{4} A_{i} \cdot \sum_{j \neq i} E_{j} & =-\left(E_{3}^{0}+E_{4}^{0}+\varepsilon_{2}-i \gamma\right) \\
\sum_{i=1}^{4} A_{i} \cdot \sum_{k \neq l \neq i} E_{k} \cdot E_{l} & =E_{3}^{0} E_{4}^{0}+\left(\varepsilon_{2}-i \gamma\right)\left(E_{3}^{0}+E_{4}^{0}\right) \\
\sum_{i=1}^{4} A_{i} \cdot \prod_{j \neq i}^{j} E_{j} & =-\left(\varepsilon_{2}-i \gamma\right) E_{3}^{0} E_{4}^{0}
\end{aligned}
$$

$E_{i}^{0}$ - are the eigenvalues of equation (15) with electronphonon coupling constant $g=0$.

$$
\begin{aligned}
& E_{1,2}^{0}=E_{1,2} \\
& E_{3,4}^{0}=\omega_{0}+E_{1,2}
\end{aligned}
$$

Equation for Keldysh Green function $G_{11}^{<}\left(t, t^{\prime}\right)$, which determines localized charge time evolution $n_{1}(t)$ than has the form:

$$
\begin{array}{r}
G_{11}^{<}\left(t, t^{\prime}\right)=G_{11}^{0<}+G_{11}^{0 R} T^{2} G_{22}^{0 R} G_{11}^{<}+ \\
+G_{11}^{0 R} T^{2} G_{22}^{0<} G_{11}^{A}+G_{11}^{0<} T^{2} G_{22}^{0 A} G_{11}^{A}+ \\
+G_{11}^{0 R} \Sigma_{11}^{<} G_{11}^{A}+G_{11}^{0 R} \Sigma_{11}^{R} G_{11}^{<}+G_{11}^{0<} \Sigma_{11}^{A} G_{11}^{A}
\end{array}
$$

where self-energy $\Sigma_{11}^{<}\left(t, t^{\prime}\right)$ can be written as:

$$
\Sigma_{11}^{<}\left(t, t^{\prime}\right)=i g^{2} D^{<}\left(t, t^{\prime}\right) G_{22}^{<}\left(t, t^{\prime}\right)
$$

with phonon function $D^{<}\left(t_{1}, t_{2}\right)$ :

$D^{<}\left(t_{1}, t_{2}\right)=-i\left(N_{\omega 0}+1\right) e^{-i \omega_{0}\left(t_{1}-t_{2}\right)}-i N_{-\omega 0} e^{i \omega_{0}\left(t_{1}-t_{2}\right)}$

acting with operator $G_{11}^{0 R-1}$ equation (20) can be rewritten in the following form:

$$
\begin{array}{r}
G_{11}^{0 R-1} G_{11}^{<}\left(t, t^{\prime}\right)=\left(i \frac{\partial}{\partial t}-\varepsilon_{1}\right) G_{11}^{<}\left(t, t^{\prime}\right)= \\
=T^{2} \int_{0}^{\infty} d t_{1} \cdot G_{22}^{0 R}\left(t, t_{1}\right) G_{11}^{<}\left(t_{1}, t^{\prime}\right)+ \\
+\int d t_{1}\left[\Sigma_{11}^{<}\left(t, t_{1}\right) G_{11}^{A}\left(t_{1}, t^{\prime}\right)+\Sigma_{11}^{R}\left(t, t_{1}\right) G_{11}^{<}\left(t_{1}, t^{\prime}\right)\right]
\end{array}
$$

Green's function $G_{11}^{<}\left(t, t^{\prime}\right)=i n_{1}(t)$ is determined by the sum of homogeneous and inhomogeneous solutions:

$$
\begin{array}{r}
n_{1}(t)=n_{1}^{h}(t)+\widetilde{n}_{1}(t)=n_{1}^{h}(t)+ \\
+\int_{0}^{t} G_{11}^{R}\left(t, t_{1}\right) \Sigma_{11}^{<}\left(t, t_{2}\right) G_{11}^{A}\left(t_{2}, t\right) d t_{1} d t_{2}
\end{array}
$$

Homogeneous solution of the equation can be written in the following way:

$$
n_{1}^{h}(t)=n_{1}^{0} \cdot \sum_{i j} A_{i} A_{j}^{*} e^{-i\left(E_{i}-E_{j}^{*}\right) t}
$$

where coefficients $A_{i}$ correspond to the Green's function $G_{11}^{R}$, which is determined by the equation (17). Function $G_{22}^{<T}\left(t_{1}, t_{2}\right)$ can be written as:

$$
G_{22}^{<T}\left(t_{1}, t_{2}\right)=\sum_{i^{\prime} j^{\prime}=1,2} a_{i^{\prime} j^{\prime}} e^{-i E_{i^{\prime}}^{0} t_{1}} \cdot e^{i E_{j^{\prime}}^{* 0} t_{2}}
$$


and coefficients $a_{i^{\prime} j^{\prime}}$ have the following form:

$$
\begin{aligned}
& a_{11}=a_{22}=\frac{i T^{2}}{\left|E_{2}^{0}-E_{1}^{0}\right|^{2}} \\
& a_{12}=a_{21}^{*}=-a_{11}
\end{aligned}
$$

Consequently one can find the inhomogeneous solution of the equation

$$
\begin{array}{r}
\widetilde{n}_{1}(t)=g^{2} \sum_{i j i^{\prime} j^{\prime}=1}^{4} A_{i} \cdot A_{j}^{*} \cdot a_{i^{\prime} j^{\prime}} . \\
\frac{-1}{i\left(E_{j}^{*}-E_{j^{\prime}}^{0 *}-\omega_{0}\right)} \cdot \frac{1}{i\left(E_{i}-E_{i^{\prime}}^{0}-\omega_{0}\right)} \cdot \\
\cdot\left[e^{-i\left(E_{i^{\prime}}^{0}+\omega_{0}\right) t}-e^{-i E_{i} t}\right]\left[e^{i\left(E_{j^{\prime}}^{0 *}+\omega_{0}\right) t}-e^{i E_{j}^{*} t}\right]
\end{array}
$$

Considering only the leading terms in parameters $\frac{g^{2}}{\omega_{0}^{2}}$, $\frac{T^{2}}{\gamma^{2}}$ in eq.(28), the following expression for the inhomogeneous solution can be obtained:

$$
\begin{array}{r}
\tilde{n}_{1}(t)=\frac{g^{2}}{\omega_{0}^{2}} \cdot \frac{T^{2}}{\gamma^{2}} \sum_{i^{\prime} j^{\prime}=1}^{2}\left[e^{-i\left(E_{i^{\prime}}^{0}+\omega_{0}\right) t}-e^{-i E_{1} t}\right] . \\
\cdot\left[e^{i\left(E_{j^{\prime}}^{0 *}+\omega_{0}\right) t}-e^{i E_{1}^{*} t}\right]
\end{array}
$$

\section{RESULTS AND DISCUSSION}

The behavior of filling numbers time evolution depends on the parameters of the system: energy levels detuning, the relation between tunneling rates and electron-phonon coupling constant, the value of optical phonon frequency. The general feature of all dependencies is the increasing of localized charge relaxation rate caused by the electronphonon interaction.

We start by discussing the filling numbers time evolution in the case of the positive initial detuning between energy levels in the coupled QDs $\left(\Delta \varepsilon=\varepsilon_{1}-\varepsilon_{2}>0\right)$. Obtained calculation results are presented on the Fig 1, It is clearly evident that electron-phonon interaction leads to the increasing of localized charge relaxation rate. The growth of the electron-phonon coupling constant $g$ for a given set of system parameters results in the increasing of filling numbers relaxation rate. With the increasing of the initial detuning the influence of electron-phonon interaction on the charge time evolution is clearly pronounced (see Fig[1]).

A critical value of the detuning exists in the system under investigation for a given set of parameters which corresponds to the relaxation regime changing. For the smaller values of the detuning charge relaxation takes place with the only one relaxation rate both in the presence (see grey and black-dashed lines on the Fig,1b) and
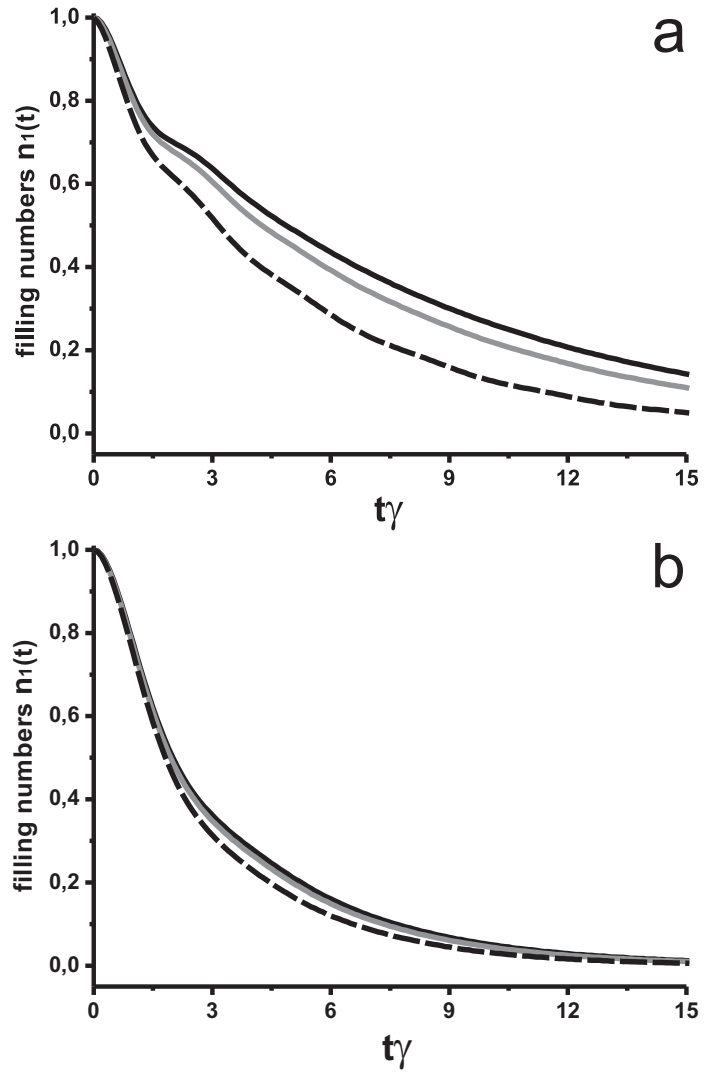

FIG. 1: Fig.1 Filling numbers time evolution in the presence of electron-phonon interaction in the case of positive initial detuning $\Delta \varepsilon$. Black line corresponds to the case when $g=0$, grey line describes the situation when $g=0.1$ and blackdashed line - $g=0,2$. a) $\Delta \varepsilon=2,0, \omega_{0}=2,0$; b) $\Delta \varepsilon=1,0$, $\omega_{0}=1,0$. For all the figures values of parameters $T=0,6$, $\gamma=1,0$ are the same.

in the absence of electron-phonon interaction (see black line on the Fig 10). The typical time scale which determines the localized charge relaxation is close to the value $\gamma_{\text {res }}=2 \frac{T^{2}}{\gamma}$. For the larger values of detuning than the critical one, localized charge time evolution reveals two typical time intervals with different values of the relaxation rates (see Fig 1a). The first time interval relaxation rate exceeds the relaxation rate of the second time interval both in the presence and in the absence of electron-phonon coupling. Without electron-phonon interaction the first time interval reveals charge relaxation with the typical rate $\gamma_{\text {res }}$. The second time interval demonstrates charge time evolution with relaxation rate close to $\gamma_{\text {nonres }}=\gamma_{\text {res }} \frac{\gamma^{2}}{\Delta \varepsilon^{2}}$. When electron phononcoupling is involved, the filling numbers time evolution in the first time interval has the value $\gamma \sim 2 \gamma_{\text {res }}$ and in the second time interval $-\gamma \sim 2 \gamma_{\text {nonres }}$. Consequently, electron-phonon interaction results in the two times increasing of localized charge relaxation rate.

Let us now focus on the charge relaxation processes in the case of negative initial detuning between energy levels in the QDs $\left(\varepsilon_{1}<\varepsilon_{2}\right)$ (see Fig 2 ). One can clearly see 

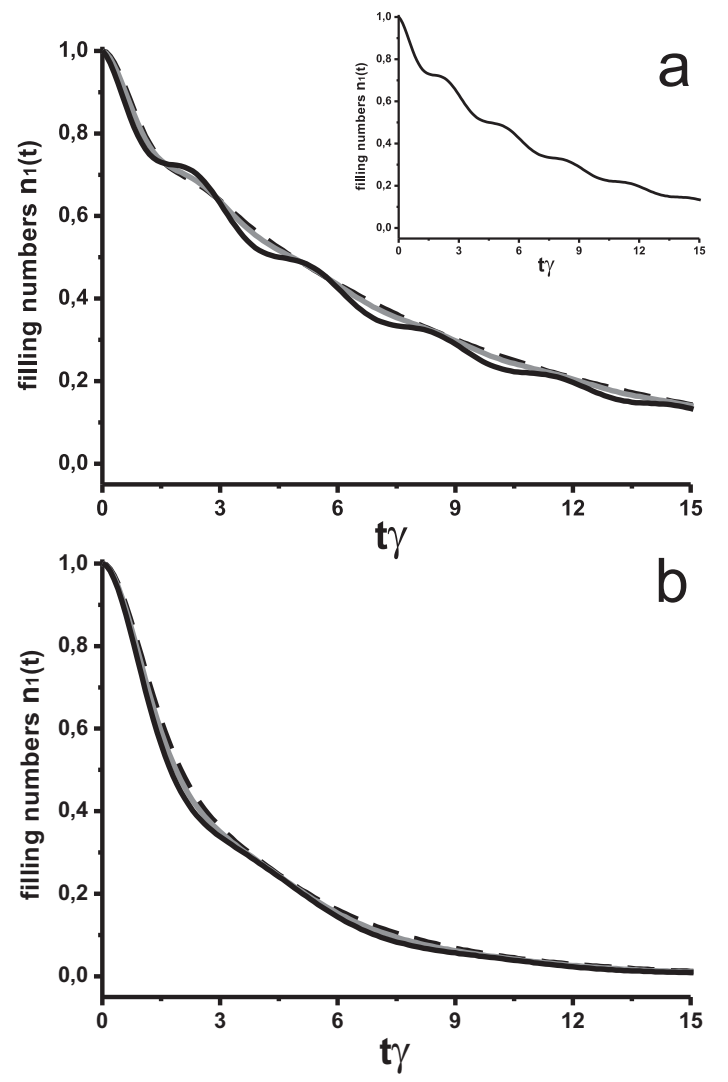

FIG. 2: Fig.2 Filling numbers time evolution in the presence of electron-phonon interaction in the case of negative initial detuning $\Delta \varepsilon$. Black-dashed line corresponds to the case when $g=0$, grey line describes the situation when $g=0.1$ and black line - $g=0,2$. a) $\Delta \varepsilon=-2,0, \omega_{0}=2,0$; b) $\Delta \varepsilon=$ $-1,0, \omega_{0}=1,0$. For all the figures values of parameters $T=0,6, \gamma=1,0$ are the same. The inset demonstrates localized charge relaxation in the case when $g=0,2$.

that in the case of negative detuning electron-phonon interaction also results in the increasing of localized charge relaxation rate, but this effect is less pronounced. Charge time evolution changes slightly in comparison with the situation when positive detuning occurs. In the case of negative detuning always exist several time intervals with different values of the relaxation rates (see Fig 2). For the small value of initial detuning (see Fig 2 b) relaxation rates on the both time intervals are very close to each other and to the value $\gamma_{\text {res }}$ both in the presence and in the absence of electron-phonon interaction.
For the larger value of initial detuning in the case when electron-phonon coupling is absent the first time interval reveals charge relaxation with the typical rate $\gamma_{\text {res }}$ and the second time interval corresponds to the relaxation rate $\gamma_{\text {nonres }}$. When electron-phonon coupling is considered, relaxation rates increase slightly and they are continue being very close o the values $\gamma_{\text {res }}$ and $\gamma_{\text {nonres }}$ for the first and second time intervals correspondingly.

The most interesting effect in this energy levels configuration is the formation of oscillations in the filling numbers time evolution, caused by the emission and adsorption of phonons by the energy levels when detuning is close to the phonon frequency. Oscillations are well pronounced for the large value of initial detuning (see Fig $\sqrt{2} \mathrm{a}$ and the inset). For a given set of system parameters the oscillations amplitude is determined by the value of the electron-phonon coupling constant $g$ (see Fig 2). The presence of oscillations may even lead to the decreasing of filling numbers relaxation rate in comparison with the case when electron-phonon interaction is absent (see black-dashed and black lines on the Fig $2 \mathrm{a}$ ) for a given set of system parameters in the particular time intervals.

\section{CONCLUSION}

We investigated filling numbers time evolution in the system of two interacting QDs weakly coupled to the reservoir in the presence of electron-phonon interaction. It was shown that electron-phonon interaction results in the increasing of localized charge relaxation rate. The value of extantion is determined by the system parameters such as energy levels detuning, optical phonon frequency and the ratio between electron-phonon coupling constant and tunneling transfer amplitudes.

We revealed that in the case of positive initial detuning between energy levels in the dots the influence of electron-phonon interaction is mostly pronounced and the relaxation rate increases with the growth of the initial detuning value.

We found that when negative initial detuning is considered, the influence of electron-phonon interaction leads to the formation of oscillations in the filling numbers time evolution. These oscillations are the result of phonons emission and adsorption between the energy levels.

This work was supported by RFBR grants and by the National Grants of Ministry of science and education.
[1] K.Y. Tan, K.W. Chan, M. Mottonen, et.al., Nano Lett., 10, 11 (2010).

[2] K. Grove-Rasmussen, H.I. Jorgensen, T.Hayashi, et.al., Nano Lett., 8, 1055 (2008).

[3] D. Loss, D.P. DiVincenzo, Phys. Rev. A, 57, 120 (1998).

[4] P.I. Arseyev, N.S. Maslova, V.N. Mantsevich, JETP Letters, 95(10), 521 (2012).
[5] P.I. Arseyev, N.S. Maslova, V.N. Mantsevich, European Physical Journal B, 85(7), 249 (2012).

[6] V.N. Mantsevich, N.S. Maslova, and P.I. Arseyev, Solid State Comm., 152, 1545 (2012).

[7] L.D. Contreras-Pulido, J. Splettstoesser, M. Governale, et.al., Phys. Rev. B, 85, 075301 (2012).

[8] F. Comas, N. Studart, Phys. Rev. B, 69, 235321 (2004). 
[9] M. Keil, H. Schoeller, Phys. Rev. B, 66, 155314 (2002).

[10] J.X. Zhu, A.V. Balatsky, Phys. Rev. B, 67, 165326 (2003).

[11] B. Dong, H.L. Cui, X.L. Lei, et.al., Phys. Rev. B, 71, 045331 (2005).

[12] U. Bockelmann, Phys. Rev. B, 50, 17271 (1994).

[13] Z.J. Wu, K.D. Zhu, X.J. Yuan, et.al., Phys. Rev. B, 71, 205323 (2005).

[14] V.N. Stavrou, X. Hu, Phys. Rev. B, 72, 075362 (2005).
[15] J.I. Climente, A. Bertoni, G. Goldoni, et.al., Phys. Rev. $B, \mathbf{7 4}, 035313$ (2006).

[16] T. Fujisawa, D.G. Austing, Y. Tokura, et.al., J. Phys: Condensed Matter, 15, R1395 (2003).

[17] J.L. Cheng, M.W. Wu, C. Lu, Phys. Rev. B, 69, 115318 (2004).

[18] L.V. Keldysh, Sov. Phys. JETP, 20, 1018 (1964). 